\title{
Prospective study to evaluate the prognostic value of different nutritional assessment scores in liver surgery: NURIMAS Liver (DRKS00006340)
}

\author{
Pascal Probst ${ }^{1}$, Juri Fuchs ${ }^{1}$, Michael R. Schön ${ }^{2}$, Georgios Polychronidis ${ }^{1}$, Christos Stravodimos ${ }^{2}$, \\ Arianeb Mehrabi ${ }^{1}$, Markus K. Diener ${ }^{1}$, Philipp Knebel ${ }^{1}$, Markus W. Büchler ${ }^{1}$, Katrin Hoffmann ${ }^{1}$ \\ ${ }^{1}$ Department of General, Visceral and Transplantation Surgery, University of Heidelberg, Im Neuenheimer Feld 110, 69120 Heidelberg, Germany; \\ ${ }^{2}$ Department of General and Visceral Surgery, Städtisches Klinikum Karlsruhe, Moltkestraße 90, 76133 Karlsruhe, Germany \\ Contributions: (I) Conception and design: P Probst, J Fuchs, MR Schoen, G Polychronidis, A Mehrabi, MK Diener, P Knebel, K Hoffmann; \\ (II) Administrative support: P Probst, J Fuchs, MR Schoen, G Polychronidis, C Stravodimos, A Mehrabi, MK Diener, P Knebel, MW Büchler, \\ K Hoffmann; (III) Provision of study material or patients: All authors; (IV) Collection and assembly of data: P Probst, J Fuchs, MR Schoen, G \\ Polychronidis, C Stravodimos, MK Diener, P Knebel, MW Büchler, K Hoffmann; (V) Data analysis and interpretation: P Probst, J Fuchs, K \\ Hoffmann; (VI) Manuscript writing: All authors; (VII) Final approval of manuscript: All authors. \\ Correspondence to: Pascal Probst. Department of General, Visceral, and Transplantation Surgery, University of Heidelberg, Im Neuenheimer Feld 110, \\ 69120 Heidelberg, Germany. Email: pascal.probst@med.uni-heidelberg.de.
}

Background: Malnutrition is recognised as a preoperative risk factor for patients undergoing hepatic resection. It is important to identify malnourished patients and take preventive therapeutic action before surgery. However, there is no evidence regarding which existing nutritional assessment score (NAS) is best suited to predict outcomes of liver surgery.

Methods: All patients scheduled for elective liver resection at the surgical department of the University Hospital of Heidelberg and the Municipal Hospital of Karlsruhe were screened for eligibility. Twelve NASs were calculated before operation, and patients were categorised according to each score as being either at risk or not at risk for malnutrition. The association of malnutrition according to each score and occurrence of at least one major complication was the primary endpoint, which was achieved using a multivariate logistic regression analysis including established risk factors in liver surgery as covariates.

Results: The population consisted of 182 patients. The percentage of patients deemed malnourished by the NAS varied among the different scores, with the lowest being 2.20\% (Mini Nutritional Assessment) and the highest 52.20\% (Nutritional Risk Classification). Forty patients (22.0\%) had a major complication. None of the scores were significantly associated with major complications.

Conclusions: None of the twelve investigated NAS defined a state of malnutrition that was independently associated with postoperative complications. Other means of measuring malnutrition in liver surgery should be investigated prospectively.

Keywords: Malnutrition; liver surgery; hepatic resection; nutritional assessment

Submitted Mar 04, 2019. Accepted for publication Jun 11, 2019.

doi: 10.21037/hbsn.2019.06.11

View this article at: http://dx.doi.org/10.21037/hbsn.2019.06.11

\section{Introduction}

Malnutrition is often regarded as one of the key factors in perioperative patient management. Many studies suggest that poor nutritional status before surgery increases the risk of postoperative morbidity and mortality (1-3). For patients undergoing liver resection, nutritional status is considered particularly relevant $(4,5)$. One of the predictive variables for successful outcome after liver resection is the remnant liver's 
ability to regenerate and its capacity to sustain metabolic, synthesising, and detoxifying functions $(4,6)$. Therefore, nutritional status before surgery is regarded as one of the vital variables influencing this process (7-9). However, few studies investigate which specific screening instruments for malnutrition are suited to predict postoperative liver failure (PLF) and other complications after hepatic resection.

In the past, different approaches have been investigated to define a patient's preoperative nutritional status and to find predictive nutritional markers for complications after liver resection $(8,10-13)$. Since single markers have not proven to be reliable, nutritional assessment scores (NAS) with aggregated information were introduced to facilitate the quick identification of patients at risk for malnutrition. A systematic review by van Bokhorst-de van der Schueren et al. investigated the validity and the predictive value of 32 scores (14), showing that only a few scores have been comprehensively validated, but not in a specific surgical context. However, they also considered developing new scores to be redundant and were not hopeful that doing so would produce scores of a higher quality. The authors rather called for prospective trials investigating nutritional scores in different patient populations. Following this recommendation, the Nutritional Risk in Major Abdominal Surgery (NURIMAS) studies were started. This is a series of studies comparing different nutritional scores in specific patient populations. A trial with patients undergoing pancreatic surgery, NURIMAS Pancreas (15), has already been conducted. The aim of the NURIMAS Liver trial is therefore to identify the clinical NAS that is most suitable for predicting postoperative complications in patients undergoing liver surgery.

We present the following article in accordance with the TREND reporting checklist (available at http://dx.doi. org/10.21037/hbsn.2019.06.11.

\section{Methods}

Nutritional Risk in Major Abdominal Surgery (NURIMAS) Liver is a bicentric, prospective observational trial. The department of surgery at the University Hospital of Heidelberg served as the primary center, while a smaller portion of the population was investigated in the Municipal Hospital of Karlsruhe. Both centers have been certified for liver surgery by the German Society of General and Visceral Surgery. The trial was conducted in accordance with the Declaration of Helsinki in its current version (16) and was registered with the German Clinical Trials Register before the first patient was enrolled (DRKS00010923). As provided in the professional code for physicians in Germany $(\$ 15$ BOÄ), the trial protocol was reviewed and approved by the Ethics Committee of the medical faculty of the University of Heidelberg (S336-2016) and the Ethics Committee of the State Chamber of Physicians of BadenWürttemberg (B-F-2017-059). Additionally, the trial protocol was published open access (17).

\section{Eligibility criteria and patient recruitment}

All patients between ages 18 and 99 who were scheduled for elective liver resections at the Department of General, Visceral, and Transplantation Surgery at the University of Heidelberg or the Municipal Hospital of Karlsruhe were screened for eligibility. The only exclusion criterion was a history of liver resection; all underlying diseases were included. Eligible patients were informed about the background, purpose, and procedures associated with the trial the day before their operation, and were required to give written informed consent before being enrolled.

\section{Trial flow and clinical pathway}

After giving consent to participate in the trial, patients went through a physical examination by a medical professional trained in nutritional medicine and filled in a questionnaire including all information necessary for the 12 NASs. All assessments took place less than 48 hours prior to surgery. Additionally, information on established risk factors for postoperative complications in liver surgery were collected (18-22).

After the surgical procedure, it was first evaluated whether or not the operation was in line with the trial criteria, i.e., that a liver resection had been performed. Afterwards, the postoperative course was tracked throughout the hospital stay. The first visit took place between postoperative day (POD) 3 and 7, the second between POD 10 and 14, and a third one on the day of discharge or POD 30, whichever occurred later. Patients who were readmitted to the hospital with postoperative complications or transferred to another medical institution were followed up until final discharge.

All patients with similar kinds of resection were assigned to a standardised clinical pathway based on internal guidelines. Patients with hemihepatectomy, mesohepatectomy, or trisectionectomy usually spent 6 hours in the recovery room before they were moved to the normal ward after surgery. When vascular reconstructions were performed or extensive 
blood loss occurred during the operation, patients spent the first night in intensive or intermediate care. Patients with minor resections were usually moved to the normal ward 4 hours after the procedure. Beginning on POD 1, a full oral diet was gradually resumed. Patients were mobilised as early as possible and had one session of physiotherapy each day, as of POD 1.

\section{Sample size calculation}

Calculations of sample size in diagnostic studies differs from those in interventional trials. The method reported by Carley et al. (23) was used with the following parameters: The prevalence of malnutrition in liver surgery patients has been estimated at about $60 \%$, based on the numbers reported in various studies investigating malnutrition rates $(8,24-26)$. Based on a specificity and sensitivity of $95 \%$ and a confidence interval of 0.05 , a total of 180 patients had to be analysed.

\section{NAS (diagnostic intervention)}

Eleven of the 12 NAS that were tested in this trial were selected based on a meta-analysis by van Bokhorst-de van der Schueren (14). In this systematic review, the authors compared 32 NAS for their construct and/or criterion validity, as well as their predictive value for clinical outcomes. Scores that seemed promising, needed further validation, and fit a surgical population were chosen: Nutritional Risk Index (NRI), Nutrition Risk Score (NRS), Nutritional Risk Screening (NRS 2002), Subjective Global Assessment (SGA), Malnutrition Universal Screening Tool (MUST), Mini Nutritional Assessment (MNA and MNASF), Short Nutritional Assessment Questionnaire (SNAQ), Imperial Nutritional Screening System (INSYST I and INSYST II), Nutritional Risk Classification (NRC) (27-36). Additionally, the latest version of the European Society for Clinical Nutrition and Metabolism (ESPEN) malnutrition criteria was investigated (37). Detailed information on the investigated NAS is available in the NURIMAS Pancreas trial (15). Since NURIMAS Liver is a validation study, the cut-offs for malnutrition as proposed in the original publications of each NAS were used.

\section{Study endpoints}

Primary endpoint: postoperative morbidity and mortality Any deviation from the standardised postoperative course was assessed during the study visits. Validated definitions of liver surgery specific complications were applied (38-41). All complications were graded according to the validated Clavien-Dindo classification (42). For each score, the association between the presence of malnutrition and occurrence of at least one major complication (ClavienDindo III/IV and mortality) was analysed using a multivariate logistic regression model as described below.

\section{Secondary endpoints}

As secondary endpoints, the comprehensive complication index (CCI) (43), length of hospital stay, length of stay in the intensive care unit, administration of postoperative enteral or parenteral nutrition, and place of discharge (whether to home, a rehabilitation or care facility, or another hospital/department) of patients who were deemed at risk of malnutrition according to the NAS were compared with those who were not at risk.

\section{Methods for minimising bias}

To avoid selection bias, all patients admitted to the trial sites were screened consecutively for eligibility. Everyone who met inclusion criteria and gave informed consent to the trial was included in this single-arm trial. To minimise performance and detection bias, no analyses, neither of pre- nor postoperative data, were performed before the trial ended. Since data for the different NAS were collected in an aggregate form, it was not possible to see whether a patient was rated as being "at risk for malnutrition" by any of the scores before the database was closed. Thus, the postoperative course was followed without bias and without knowing a patient's nutritional status as assessed by the NAS. While surgeons knew about the patient's participation in the trial, they were blinded concerning the result of the nutritional assessment. By publishing a protocol with detailed information about the trial (17) and registering the trial in the German Clinical Trial Register (DRKS00010923), selective reporting was prevented. No statistical analyses were performed before closing the database, i.e., after the last patient's final visit had been conducted. Confounding was minimised by including covariates and cofactors in the statistical analysis of the primary endpoint.

\section{Statistical analysis}

The 12 tested NAS contain different classifications for grading the severity of malnutrition by distinguishing between two, 


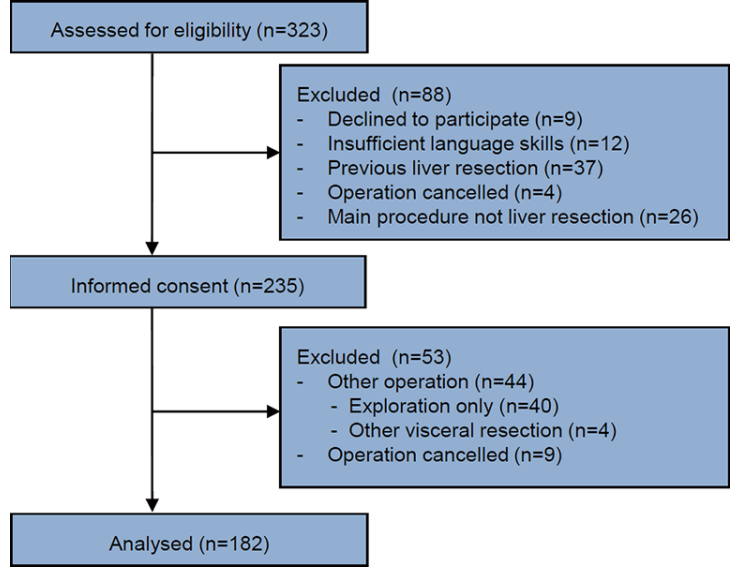

Figure 1 Flow chart of the recruitment process for NURIMAS Liver.

three, and four degrees of nutritional risk. Therefore, a simplification of the NAS-results was necessary to compare the patients' scores. Only patients in the highest nutritional risk group of each NAS were assigned the label at risk for malnutrition. Patients in all other risk categories were assigned to the not at risk for malnutrition group. For analysing the outcome variable, i.e., postoperative morbidity, patients were separated into two groups, depending on whether or not they had at least one major complication (Clavien-Dindo III/IV or mortality). For each of the scores, a diagnostic odds ratio with $95 \%$ confidence interval, sensitivity, specificity, and positive predictive value were calculated.

Two statistical tests were applied to assess the significance of the association between malnutrition and major complications for the primary endpoint. First, the association between nutritional risk and major complications was tested by using a chi-squared test with a level of significance of $5 \%$ (without Yate's-correction). Second, a multivariate logistic regression analysis was performed (level of significance $5 \%$ ) for each score. Age (years), operation time (minutes) and intraoperative blood loss $(\mathrm{mL})$ were included as covariates. Factors were HCC or Klatskin Tumor, gender, use of laparoscopy, vascular resection (portal vein, hepatic vein or artery), inclusion in an interventional liver trial, American Society of Anesthesiology (ASA) Score $\geq$ III, preoperative Serum Albumin $<35 \mathrm{~g} / \mathrm{L}$, a history of upper gastrointestinal surgery, and number of segments resected. In addition to those of the main population, all analyses were performed separately for two subgroups: Group 1 comprised all patients with resections of 3 or more liver segments (major resection). Group 2 consisted of all patients that had procedures with 2 or fewer segments resected (minor resection).

Analyses of the secondary endpoints were performed descriptively. Measures of the empirical distributions were noted in tabular form, i.e., depending on the level of variables, means, and standard deviations, or absolute and relative frequencies were given. Descriptive $\mathrm{P}$ values of the corresponding statistical test and the associated $95 \%$ confidence intervals were also entered. For all analyses, statistical computing software $\mathrm{R}$ was used (44).

\section{Results}

Patient recruitment took place between August 2016 and February 2018. A total of 323 patients were screened for eligibility. 235 were included in the trial, with causes for exclusion as follows: previous liver resection (37), main procedure not a liver resection $(\mathrm{n}=26)$, insufficient German language skills $(n=12)$, refusal to participate $(n=9)$, and cancellation of operation prior to informed consent $(n=4)$. Of these 235 patients, a total of 182 were finally analysed. Among those patients excluded from analysis due to operations other than liver resection, 40 of these were exploratory, 4 were other visceral resections and 9 cancellation of operation after informed consent. A flow chart of the recruitment process is shown in Figure 1. Of the 182 patients analysed, 55 received an atypical liver resection. Anatomical resection was performed in 127 patients: among these were 32 right hemihepatecomies and 14 right trisectionectomies, 21 left hemihepatecomies and 3 left trisectionectomies, and 57 other anatomical resections.

Table 1 gives an overview of the baseline characteristics of the main population as well as the two subgroups of the trial population for better comparability. The main population comprised 76 females (41.8\%) and 106 males (58.2\%). Mean body mass index (BMI) was $26.8 \pm 5.5 \mathrm{~kg} / \mathrm{m}^{2}$. Weight loss occurred in 66 patients, with a mean loss of $2.1 \mathrm{~kg}$ or $2.7 \%$ of the original weight.

The proportion of patients rated as at risk of malnutrition ranged from $2.20 \%$ (SGA) to $52.20 \%$ (NRC). Figure 2 shows patients labelled at risk according to all 12 NAS. A major complication (Clavien-Dindo III-V) occurred in 40 of 182 patients $(22.0 \%)$, including 5 postoperative deaths (2.8\%). Table 2 shows detailed information on postoperative mortality and morbidity.

\section{Primary endpoint}

The association between nutritional risk according to the 
Table 1 Baseline patient characteristics

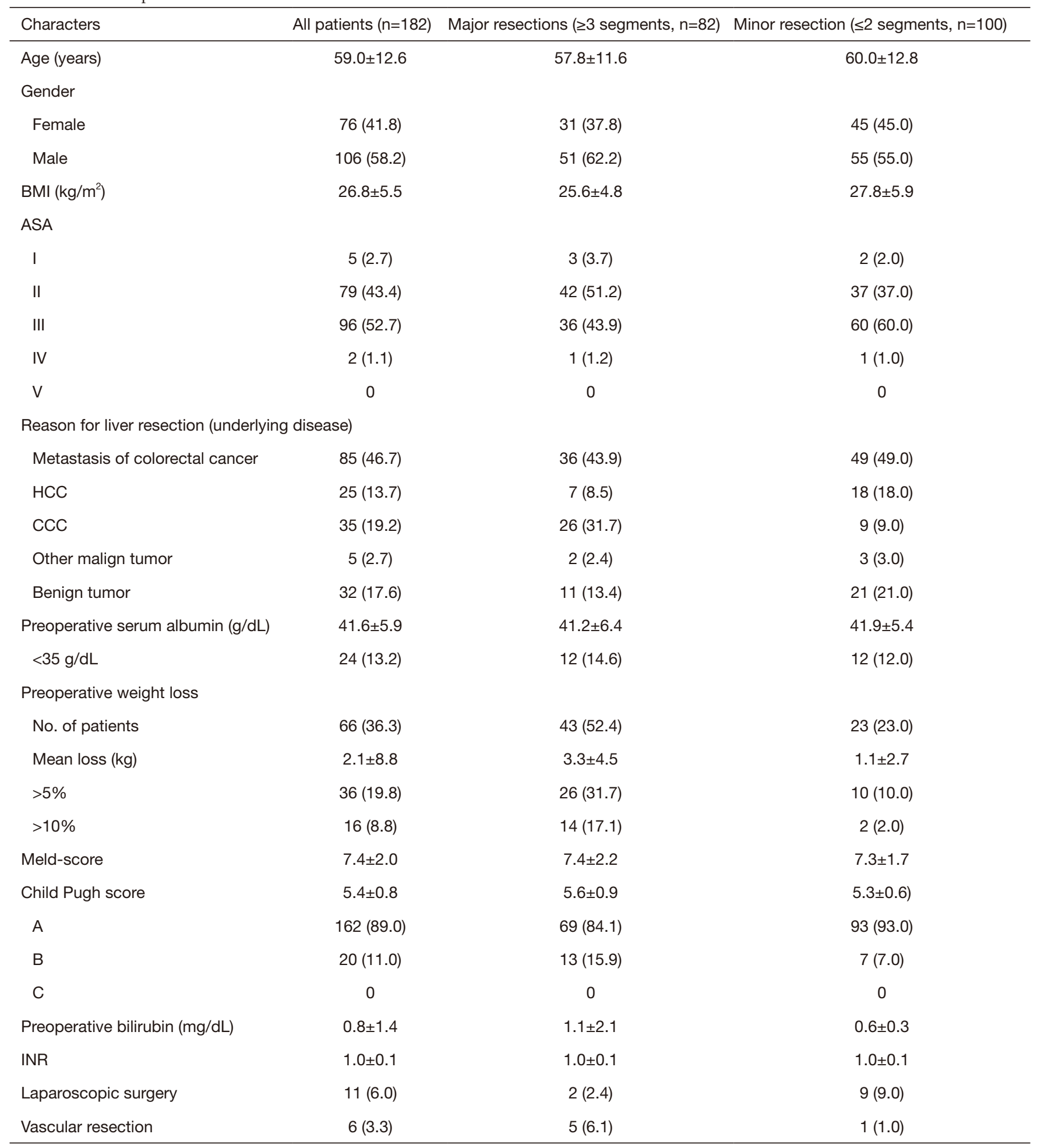

Data are shown as mean \pm standard deviation or number (percent). BMI, body mass index; ASA, American Society of Anesthesiology. 


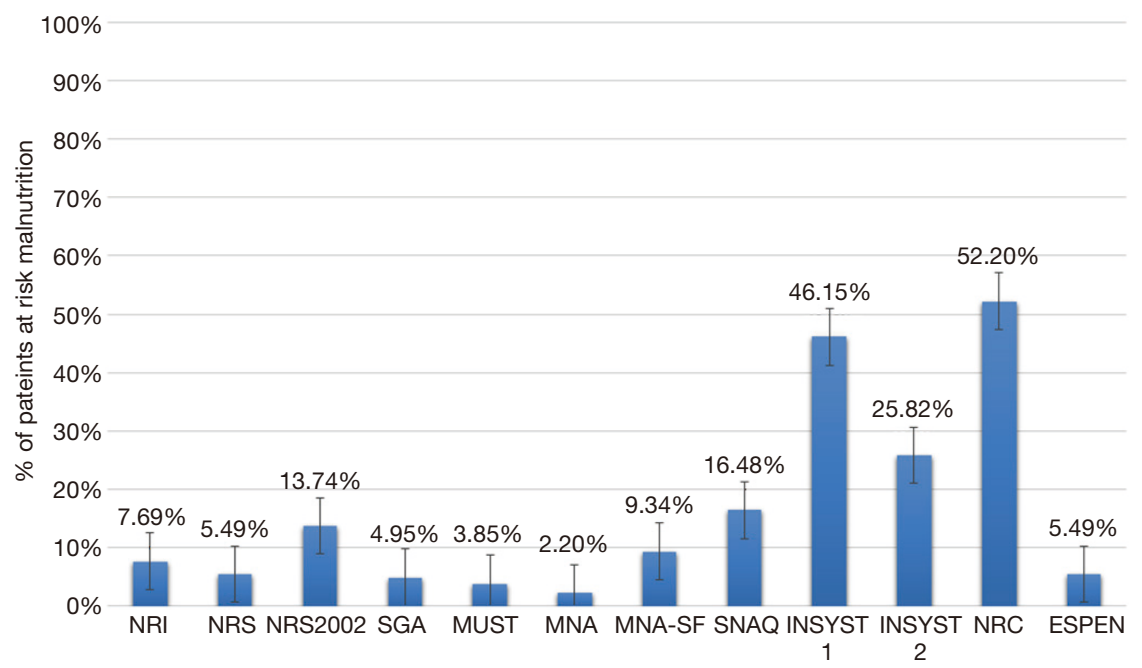

Figure 2 Proportion of patients at risk of malnutrition according to the different nutritional assessment scores. Error bars represent $95 \%$ confidence intervals. NRI, Nutritional Risk Index; NRS, Nutritional Risk Screening Score; SGA, Subjective Global Assessment; MUST, Malnutrition Universal Screening Tool; MNA, Mini Nutritional Assessment; MNA-SF, Mini Nutritional Assessment Short Form; SNAQ, Short Nutritional Assessment Questionnaire; INSYST, Imperial Nutritional Screening System; NRC, Nutrition Risk Classification; ESPEN, European Society for Clinical Nutrition and Metabolism malnutrition criteria.

Table 2 Postoperative morbidity and mortality according to the Clavien-Dindo classification

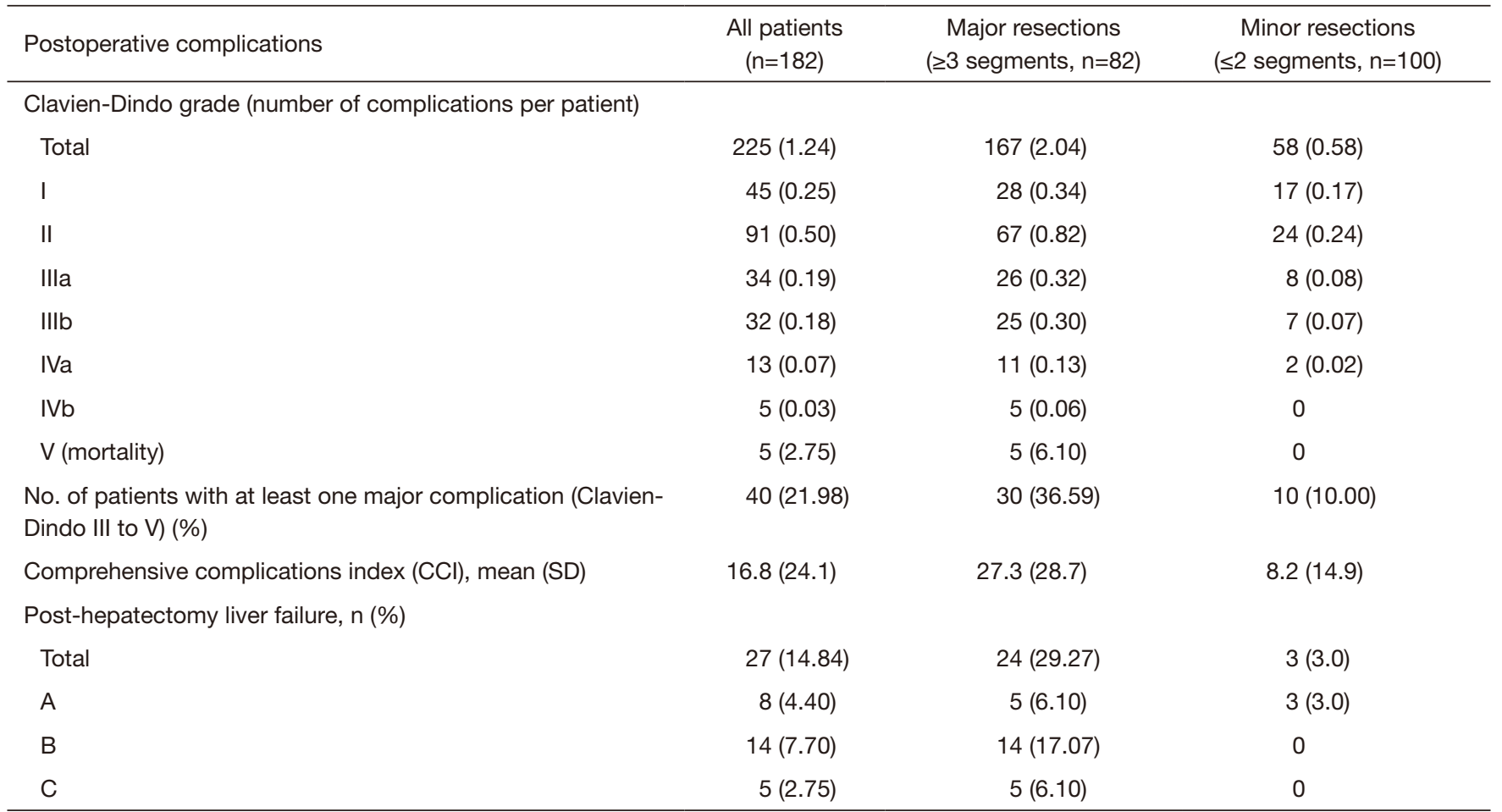

Table 2 (continued) 
Table 2 (continued)

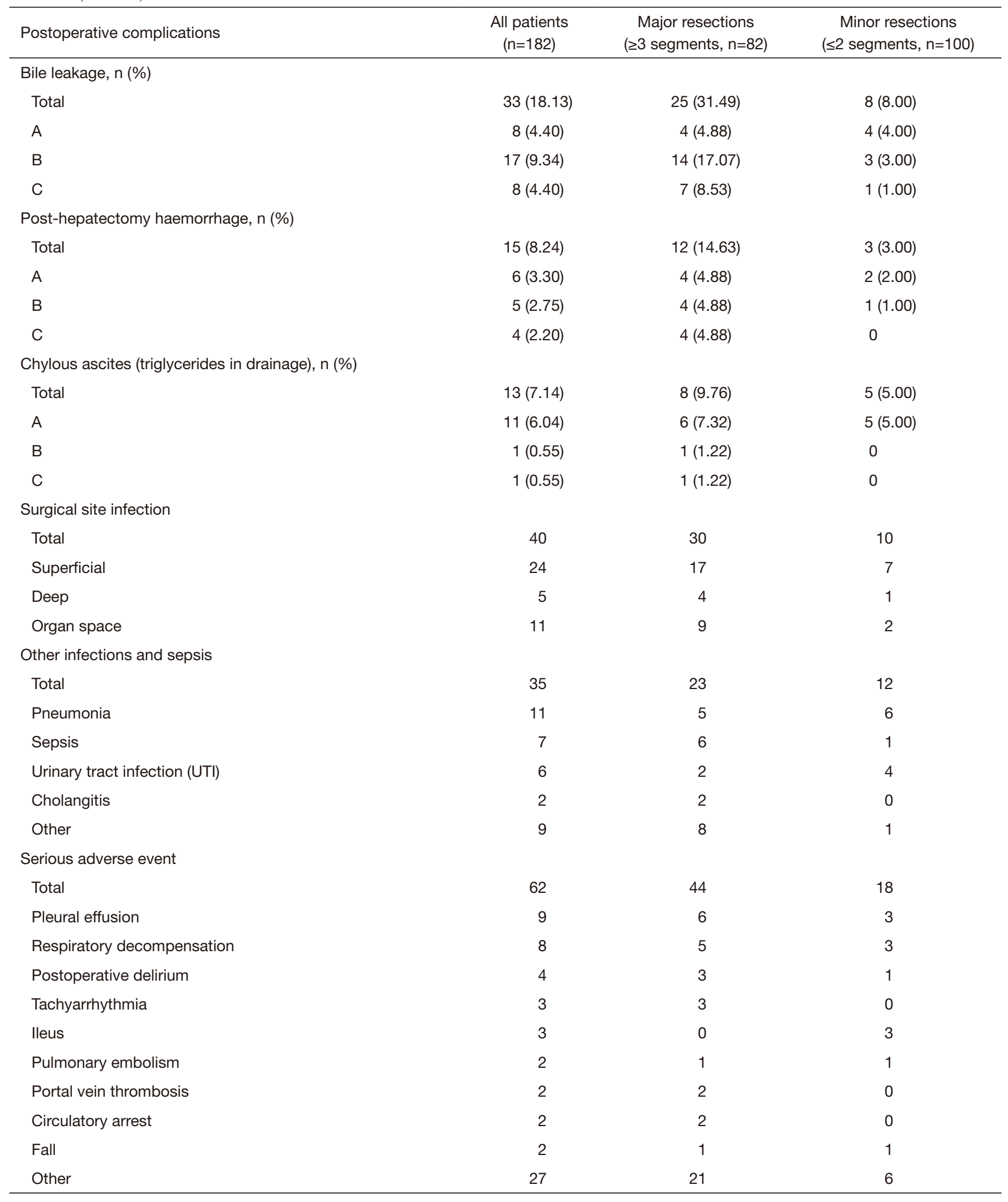


Table 3 Univariate analysis of association between risk of malnutrition estimated using different nutritional scores and major postoperative complications

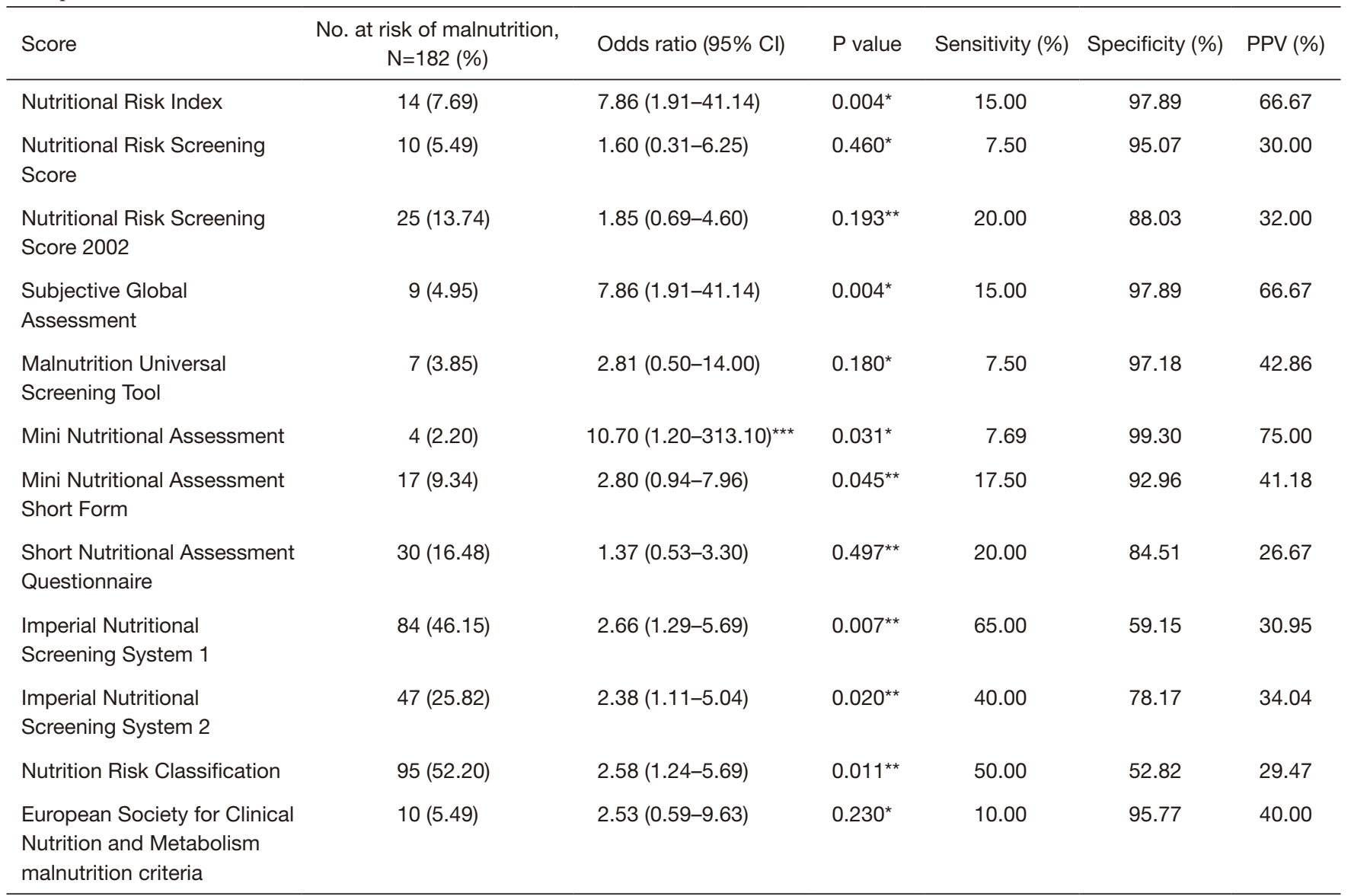

${ }^{*}$, Fisher's exact test; ${ }^{* *}$, chi-squared test; ${ }^{* \star *}$, chi-squared test correction $0 \rightarrow 1$. PPV, positive predictive value.

NAS and complications after liver resection was analysed in a univariate and multivariate calculation. Postoperative morbidity and mortality is the primary endpoint in this trial.

\section{Univariate analysis}

Within the main population, six scores had a significant association with the primary endpoint in this univariate setting, namely the NRI, SGA, MNA, INSYST1, INSYST 2, and the NRC. The MNA achieved the highest PPV, at $75.0 \%$. Sensitivity ranged from $7.5 \%$ for the NRS to $65.0 \%$ for the INSYST 1 . Specificity was between $52.8 \%$ (NRC) and $99.3 \%$ (MNA). Table 3 shows the results of the univariate analysis.

\section{Multivariate analysis}

In contrast to the univariate calculation, none of the NAS showed a significant association with the occurrence of major complications in the main population. Only the co-variable operation time proved to be independently associated with major complications ( $\mathrm{OR}=1.01,95 \% \mathrm{CI}$ : $1.00-1.01, \mathrm{P}=0.018)$. None of the other co-variables and co-factors turned out to be significant predictors. The full results of the multivariate analysis of the primary endpoint are displayed in Table 4.

In the major resection subgroup, the NRC was significantly associated with major postoperative complications ( $\mathrm{OR}=3.79,95 \% \mathrm{CI}$ : $1.06-13.49, \mathrm{P}=0.040$ ). Apart from that, only operation time had a significant association with the primary endpoint in this subgroup. No significant association was found in the multivariate analyses of the minor resection subgroup.

\section{Secondary endpoints}

\section{Comprehensive complication index}

For the whole trial population, the mean CCI was $16.8 \pm 24.1$. 
Table 4 Multivariate logistic regression analysis to determine factors associated with major complications $(n=182)$

\begin{tabular}{|c|c|c|}
\hline \multirow{2}{*}{ Target factor } & \multicolumn{2}{|c|}{ Major complication } \\
\hline & OR $(95 \% \mathrm{Cl})$ & $P$ value \\
\hline \multicolumn{3}{|l|}{ Scores } \\
\hline Nutritional Risk Index & $5.8(0.68-49.32)$ & 0.108 \\
\hline Nutritional Risk Screening Score & $1.26(0.24-6.66)$ & 0.788 \\
\hline $\begin{array}{l}\text { Nutritional Risk Screening Score } \\
2002\end{array}$ & $0.72(0.22-2.33)$ & 0.585 \\
\hline Subjective Global Assessment & $4.21(0.82-21.56)$ & 0.085 \\
\hline $\begin{array}{l}\text { Malnutrition Universal Screening } \\
\text { Tool }\end{array}$ & $1.72(0.28-10.69)$ & 0.563 \\
\hline Mini Nutritional Assessment & $6.45(0.49-85.00)$ & 0.156 \\
\hline $\begin{array}{l}\text { Mini Nutritional Assessment } \\
\text { Short Form }\end{array}$ & $1.13(0.31-4.12)$ & 0.850 \\
\hline $\begin{array}{l}\text { Short Nutritional Assessment } \\
\text { Questionnaire }\end{array}$ & $3.71(0.11-1.24)$ & 0.108 \\
\hline $\begin{array}{l}\text { Imperial Nutritional Screening } \\
\text { System } 1\end{array}$ & $1.59(0.68-3.73)$ & 0.286 \\
\hline $\begin{array}{l}\text { Imperial Nutritional Screening } \\
\text { System } 2\end{array}$ & $0.95(0.37-2.43)$ & 0.922 \\
\hline Nutrition Risk Classification & $2.11(0.85-5.26)$ & 0.110 \\
\hline $\begin{array}{l}\text { European Society for Clinical } \\
\text { Nutrition and Metabolism }\end{array}$ & $1.53(0.34-6.86)$ & 0.579 \\
\hline \multicolumn{3}{|l|}{ Co-factors } \\
\hline HCC or Klatskin & $1.72(0.51-5.77)$ & 0.384 \\
\hline Male gender & $1.11(0.41-3.03)$ & 0.831 \\
\hline Laparoscopy & Not applicable & 0.992 \\
\hline Vascular resection & $0.26(0.02-3.90)$ & 0.329 \\
\hline Interventional trial & $0.58(0.10-3.33)$ & 0.537 \\
\hline $\begin{array}{l}\text { American Society of } \\
\text { Anesthesiology } \geq 111\end{array}$ & $1.58(0.57-4.40)$ & 0.381 \\
\hline Upper gastrointestinal surgery & $1.47(0.53-4.10)$ & 0.459 \\
\hline Number of segments resected & $1.28(0.94-1.75)$ & 0.111 \\
\hline Albumin $<35 \mathrm{~g} / \mathrm{L}$ & $0.81(0.09-7.67)$ & 0.856 \\
\hline \multicolumn{3}{|l|}{ Co-variables } \\
\hline Age & $1.00(0.95-1.04)$ & 0.959 \\
\hline Operation time & $1.01(1.00-1.01)$ & 0.018 \\
\hline Intraoperative blood loss & $1.00(1.00-1.00)$ & 0.847 \\
\hline
\end{tabular}

The mean CCI for patients with at least one major complication was $54.0 \pm 22.8$, and $6.4 \pm 10.1$ for those without a major complication. In four of the scores, the CCI differed significantly in the main population between patients at risk and those not at risk of malnutrition according to the scores. These were the NRI (31.6 vs. 16.1, $\mathrm{P}=0.016$ ), the SGA (39.4 vs. $15.6, \mathrm{P}=0.025)$, the MNA (73.3 vs. 15.6, $\mathrm{P}=0.001$ ), and the INSYST II (26.4 vs. 13.5, $\mathrm{P}=0.047)$. In addition, the MNASF showed a significant difference in the subgroup major resection (45.2 vs. 24.5, $\mathrm{P}=0.034)$.

\section{Length of hospital stay (LOS)}

The median LOS was 9 days (range 2 to 68 days), with a mean of $14.2 \pm 12.7$ days. A total of 8 NAS were significantly associated with the secondary endpoint LOS, i.e., patients who were at risk of malnutrition according to the respective scores stayed in the hospital significantly longer. The NRI yielded significant results in both the main population and the subgroup major resection (27.1 vs. $13.5, \mathrm{P}=0.002$ and 35.4 vs. $18.5, \mathrm{P}=0.011$ ), as did MNA (46.0 vs. 13.5, $\mathrm{P}=0.002$ and 46.0 vs. 18.2, $\mathrm{P}=0.006)$, MNA-SF (24.0 vs. 13.2, $\mathrm{P}=0.003$ and 30.5 vs. $17.8, \mathrm{P}=0.014)$, INSYST I $(17.3$ vs. $11.5, \mathrm{P}=0.001$ and 22.0 vs. $16.0, \mathrm{P}=0.032)$ and INSYST II (20.7 vs. 11.9, $\mathrm{P}=0.001$ and 24.2 vs. $16.5, \mathrm{P}=0.023)$. The SGA for the main population (29.2 vs. 13.4, $\mathrm{P}=0.001)$, the SNAQ for the main population, and the "minor resection" subgroup (20.6 vs. 12.9, $\mathrm{P}=0.001$ and 13.6 vs. 9.5, $\mathrm{P}=0.026$ ). The NRC showed significant associations in the main population as well as both subgroups (16.9 vs. $11.3, \mathrm{P}=0.001$, 22.5 vs. $15.1, \mathrm{P}=0.021$ and 10.9 vs. $8.9, \mathrm{P}=0.028)$.

\section{Length of stay in intensive care unit}

At total of 77 of 182 patients (42.31\%) spent at least one day in intensive care. Out of the whole population, the mean stay in the ICU was $3.1 \pm 7.2$ days (range, 0-40 days). Like the endpoint LOS, a few scores showed significant results in at least one of the groups (NRI, NRS 2002, SGA, MNA, MNA-SF, SNAQ, INSYST I + II, NRC).

\section{Postoperative artificial nutrition}

Twenty patients $(11 \%)$ received parenteral or enteral nutrition during their hospitalisation. Patients at risk of malnutrition according to the following scores received artificial nutrition significantly more often (numbers for main population): $\mathrm{NRI}(\mathrm{OR}=7.7,95 \% \mathrm{CI}: 1.7-33.4)$, NRS 
(OR =6.4, 95\% CI: $1.5-25.8)$, NRS $2002(\mathrm{OR}=3.2,95 \%$ CI: 1.0-9.3), MNA (OR =25.2, 95\% CI: 2.8-749.1), MNASF $(\mathrm{OR}=8.0,95 \%$ CI: 2.5-25.1), SNAQ (OR $=4.2,95 \%$ CI: $1.5-11.5)$, INSYST I (OR $=5.3,95 \%$ CI: $1.8-19.8)$, and INSYST II (OR =5.3, 95\% CI: 2.0-14.8).

\section{Place of discharge}

All but 11 patients (6\%) were discharged to home. Five patients died, two were moved to a rehabilitation clinic, two others to a department of internal medicine, one to a surgical department, and one to a palliative care unit. INSYST II and the NRC showed an association between patients at risk of malnutrition and not being discharged to home (INSYST II: OR =3.8, 95\% CI: 1.0-14.1; NRC: OR $=8.9,95 \%$ CI: $1.6-223.8)$.

\section{Discussion}

The aim of the NURIMAS trial series is to identify the best suited NAS that is associated with postoperative complications in specific patient populations. After the first trial in the pancreatic surgery population, NURIMAS Liver investigated 12 NAS in 182 individuals who underwent hepatic resections.

The prevalence of malnutrition indicated vast differences according to the 12 NAS. NURIMAS Pancreas showed a similarly high variability among the scores (15). In the multivariate regression model, adjusted for established risk factors in hepatic surgery, none of the definitions of malnutrition according to the twelve NAS showed a significant association with major postoperative complications. Only the multivariate analysis of the subgroup of patients with resections of more than two liver segments showed a significant association with the primary endpoint for the NRC. However, twelve different scores were tested on multiple outcomes and the risk of a multiple testing bias, i.e., finding significant results by chance, must be considered. The results of the secondary endpoints were inconsistent. A few scores were significantly associated with the CCI, longer hospital stay, days in intensive care, and the necessity of artificial nutrition. The NRC showed significant results for length of hospital stay, stay in the ICU, and place of discharge; however, the NRC was not associated with the CCI.

As the NRC was the only NAS that seemed promising, this point merits a brief discussion (32). This screening tool was developed for nurses and is based on questions about recent weight loss, percentage of current weight to ideal body weight, nutritionally relevant diseases (diabetes, endstage liver disease, etc.), and food intake history (symptoms like vomiting, diarrhoea or less than half of a normal nutritional intake) (32).

Regarding the existing evidence, only four studies were found that investigated the association of NAS with short-term postoperative outcomes after liver resections $(7,10,11,45,46)$. All of these studies had a retrospective design and three of them investigated the Prognostic Nutritional Index (PNI) (47), a score that is calculated by using exclusively laboratory values (serum albumin level and total lymphocyte count) and thus might rather be an indicator of acute illness and inflammation than of nutritional status. The PNI was not investigated in NURIMAS Liver. Zacharias and Ferreira carried out a retrospective analysis of 203 liver resections and investigated the predictive value of the NRS2002 on 90-day mortality and postoperative complications (45). They showed that patients at risk of malnutrition according to the NRS2002 had a significantly higher 90-day mortality. However, it was not associated with postoperative complications. The association between the NRS 2002 and 90-day mortality could not be reproduced in the NURIMAS Liver study.

There are different possible explanations why the NASs performed poorly in this trial as opposed to previous studies that found associations between NASs and postoperative outcomes. First, it should be considered that in most of the existing studies, databases were analysed retrospectively. This brings up several possible errors that can bias results. If investigators are not blinded to the patient's nutritional status according to the scores in a retrospective setting, this could lead them to focus more on those patients at risk of malnutrition, so more complications might be recorded for them and result in a detection bias. Notably, in the publicly accessible trial protocol for NURIMAS Liver, which had been published before the analysis, all endpoints were predefined, thus preventing selective reporting. In contrast, none of the existing studies published a protocol and may thus be prone to reporting bias. However, to draw the conclusion from the presented results that malnutrition is not a relevant factor in liver resections seems unlikely and would contradict large bodies of existing evidence in this field $(4,5,9)$. Yet, a valid definition of malnutrition is still unknown and needs further high-quality studies.

Such a trial was in fact performed for pancreatic surgery patients: the first trial in this series, NURIMAS Pancreas, had a prospective design with a complete trial protocol published in advance. When considering NURIMAS 
Pancreas, one notices that most of its results were also confirmed for liver surgery by the present trial. The multivariate analysis for the main population did not show a significant association for any of the scores, which casts further doubt about the appropriateness of preoperative NAS use. However, unlike for NURIMAS Pancreas, there was one significant association in one subgroup for the NRC and a higher number of univariate significant results for the secondary endpoints. In total, 255 statistical tests were performed in NURIMAS Liver, of which 59 (21.6\%) showed a significant result, thus indicating that some real association may be present when compared to NURIMAS Pancreas, where only $4.8 \%$ of the statistical tests were significant.

The following limitation of this trial must be stated. For the subgroup analysis of major liver resections, it must be considered that only patients with resection of more than two segments were included. Definitions of major liver resections may vary throughout the literature. However, the number of resected segments was also a cofactor in the multivariable analysis of the primary endpoint. Furthermore, it must be stated that some complications like bile leaks differ between the operative procedures; however, the sample size was too small for further subgroup analyses and this should be considered for future studies. Since the 12 tested NAS use different classifications for grading the severity of malnutrition, distinguishing between two, three, or four degrees of nutritional risk, for some NAS a simplification of the results was necessary to make the patient's scores comparable. Only patients in the highest nutritional risk group of each NAS were labelled at risk for malnutrition; patients in all other risk categories were placed in the not at risk for malnutrition group. However, it should be considered that the chance of significant results would be even lower when applying a chi-squared test to a contingency table with six or eight fields compared to one with four. Simply considering all patients that are not in the lowest risk class of each score at risk also seems inappropriate, since it would dramatically increase the proportion of patients classified as malnourished and would not comply with the idea and the concept of the scores. A further limitation is that the sample size calculation was made with an estimated prevalence of malnutrition at $60 \%$ among the trial population based on different studies on prevalence in comparable settings $(8,24-26)$. Most scores indicated numbers far below this estimation and their results may thus be affected by a beta error. However, one could also assume that the true prevalence of malnutrition in patients scheduled for liver resection is in fact at $60 \%$, and that the NAS are not suited to reliably detect this condition. Alternatively, it should also be noted that a possible incidence of malnutrition defined by a score of only a few percentages, highly questions its general relevance in hepatic resections. Therefore, it was important to test these scores, even if the $60 \%$ threshold was not reached by any of the tested scores.

Given the results of NURIMAS Liver, a relevant definition and diagnosis of malnutrition in liver surgery remains yet to be determined. Possible methods that were not tested in this trial and might be more apt for assessing malnutrition include CT or MRI imaging, to determine muscle mass and diagnose sarcopenia, which has been suggested to be a negative predictor of clinical outcomes after hepatic resection (8,48-51). Future research should investigate these methods prospectively.

\section{Acknowledgments}

Established resources, facilities, and organisational structures at the University Hospital of Heidelberg were used during the conduction process. We acknowledge financial support by Deutsche Forschungsgemeinschaft within the funding programme Open Access Publishing, by the Baden-Württemberg Ministry of Science, Research and the Arts and by Ruprecht-Karls-Universität Heidelberg. The sections "background" and "methods" are based on the protocol for this trial (17).

Previous communications was presented at the Congress of the European Society for Clinical Nutrition and Metabolism, Madrid, Spain, September 2018, and the National Visceralmedicine Congress, Munich, Germany, September 2018; published as abstracts in Clinical Nutrition 2018;37:S26eS45 and Z Gastroenterol 2018;56:e351-e352. Funding: None.

\section{Footnote}

Reporting Checklist: The authors have completed the TREND reporting checklist. Available at http://dx.doi. org/10.21037/hbsn.2019.06.11

Data Sharing Statement: Available at http://dx.doi. org/10.21037/hbsn.2019.06.11

Conflicts of Interest: All authors have completed the ICMJE uniform disclosure form (available at http://dx.doi. 
org/10.21037/hbsn.2019.06.11). The authors have no conflicts of interest to declare.

Ethical Statement: The authors are accountable for all aspects of the work in ensuring that questions related to the accuracy or integrity of any part of the work are appropriately investigated and resolved. The study was conducted in accordance with the Declaration of Helsinki (as revised in 2013). As provided in the professional code for physicians in Germany ( $\$ 15$ BOÄ), the trial protocol was reviewed and approved by the Ethics Committee of the medical faculty of the University of Heidelberg (S336-2016) and the Ethics Committee of the State Chamber of Physicians of BadenWürttemberg (B-F-2017-059). Informed consent was taken from all individual participants.

Open Access Statement: This is an Open Access article distributed in accordance with the Creative Commons Attribution-NonCommercial-NoDerivs 4.0 International License (CC BY-NC-ND 4.0), which permits the noncommercial replication and distribution of the article with the strict proviso that no changes or edits are made and the original work is properly cited (including links to both the formal publication through the relevant DOI and the license). See: https://creativecommons.org/licenses/by-nc-nd/4.0/.

\section{References}

1. Leide da Silva Nunes F, Calado Ferreira Pinheiro Gadelha P, Damasceno de Souza Costa M, et al. Nutritional status and its impact on time and relocation in postoperative complications of abdominal patients undergoing surgery. Nutr Hosp 2014;30:629-35.

2. Correia MI, Waitzberg DL. The impact of malnutrition on morbidity, mortality, length of hospital stay and costs evaluated through a multivariate model analysis. Clin Nutr 2003;22:235-9.

3. Thomas MN, Kufeldt J, Kisser U, et al. Effects of malnutrition on complication rates, length of hospital stay, and revenue in elective surgical patients in the G-DRGsystem. Nutrition 2016;32:249-54.

4. Ciuni R, Biondi A, Grosso G, et al. Nutritional aspects in patient undergoing liver resection. Updates Surg 2011;63:249-52.

5. Purnak T, Yilmaz Y. Liver disease and malnutrition. Best Pract Res Clin Gastroenterol 2013;27:619-29.

6. Kishi Y, Abdalla EK, Chun YS, et al. Three hundred and one consecutive extended right hepatectomies: evaluation of outcome based on systematic liver volumetry. Ann Surg 2009;250:540-8.

7. Bo Y, Yao M, Zhang L, et al. Preoperative Nutritional Risk Index to predict postoperative survival time in primary liver cancer patients. Asia Pac J Clin Nutr 2015;24:591-7.

8. van Vledder MG, Levolger S, Ayez N, et al. Body composition and outcome in patients undergoing resection of colorectal liver metastases. Br J Surg 2012;99:550-7.

9. Schütte K, Tippelt B, Schulz C, et al. Malnutrition is a prognostic factor in patients with hepatocellular carcinoma (HCC). Clin Nutr 2015;34:1122-7.

10. Takagi K, Umeda Y, Yoshida R, et al. Preoperative Controlling Nutritional Status Score Predicts Mortality after Hepatectomy for Hepatocellular Carcinoma. Dig Surg 2019;36:226-32.

11. Okamura Y, Ashida R, Ito T, et al. Preoperative neutrophil to lymphocyte ratio and prognostic nutritional index predict overall survival after hepatectomy for hepatocellular carcinoma. World J Surg 2015;39:1501-9.

12. Voron T, Tselikas L, Pietrasz D, et al. Sarcopenia impacts on short-and long-term results of hepatectomy for hepatocellular carcinoma. Ann Surg 2015;261:1173-83.

13. Huang L, Li J, Yan JJ, et al. Prealbumin is predictive for postoperative liver insufficiency in patients undergoing liver resection. World J Gastroenterol 2012;18:7021-5.

14. van Bokhorst-de van der Schueren MA, Guaitoli PR, Jansma EP, et al. Nutrition screening tools: does one size fit all? A systematic review of screening tools for the hospital setting. Clin Nutr 2014;33:39-58.

15. Probst $\mathrm{P}$, Haller S, Bruckner T, et al. Prospective trial to evaluate the prognostic value of different nutritional assessment scores in pancreatic surgery (NURIMAS Pancreas). Br J Surg 2017;104:1053-62.

16. World Medical Association. WMA Declaration of Helsinki-Ethical principles for medical research involving human subjects. Bulletin of the World Health Organization 2001;79:373.

17. Probst P, Fuchs J, Schoen MR, et al. Nutritional risk in major abdominal surgery: NURIMAS Liver (DRKS00010923)-protocol of a prospective observational trial to evaluate the prognostic value of different nutritional scores in hepatic surgery. International Journal of Surgery Protocols 2017;6:5-10.

18. Jarnagin WR, Gonen M, Fong Y, et al. Improvement in perioperative outcome after hepatic resection: analysis of 1,803 consecutive cases over the past decade. Ann Surg 2002;236:397-406; discussion 406-7.

19. Andres A, Toso C, Moldovan B, et al. Complications of 
elective liver resections in a center with low mortality: a simple score to predict morbidity. Arch Surg 2011;146:1246-52.

20. Shimada M, Matsumata T, Akazawa K, et al. Estimation of risk of major complications after hepatic resection. Am J Surg 1994;167:399-403.

21. Vauthey JN, Pawlik TM, Ribero D, et al. Chemotherapy regimen predicts steatohepatitis and an increase in 90-day mortality after surgery for hepatic colorectal metastases. J Clin Oncol 2006;24:2065-72.

22. Sitzmann JV, Greene PS. Perioperative predictors of morbidity following hepatic resection for neoplasm. A multivariate analysis of a single surgeon experience with 105 patients. Ann Surg 1994;219:13-7.

23. Carley S, Dosman S, Jones SR, et al. Simple nomograms to calculate sample size in diagnostic studies. Emerg Med J 2005;22:180-1.

24. Read JA, Choy ST, Beale PJ, et al. Evaluation of nutritional and inflammatory status of advanced colorectal cancer patients and its correlation with survival. Nutr Cancer 2006;55:78-85.

25. Wie GA, Cho YA, Kim SY, et al. Prevalence and risk factors of malnutrition among cancer patients according to tumor location and stage in the National Cancer Center in Korea. Nutrition 2010;26:263-8.

26. Cheung K, Lee SS, Raman M. Prevalence and mechanisms of malnutrition in patients with advanced liver disease, and nutrition management strategies. Clin Gastroenterol Hepatol 2012;10:117-25.

27. Tammam JD, Gardner L, Hickson M. Validity, reliability and acceptability of the Imperial Nutritional Screening System (INSYST): a tool that does not require the body mass index. J Hum Nutr Diet 2009;22:536-44.

28. Kruizenga HM, Seidell JC, de Vet HC, et al. Development and validation of a hospital screening tool for malnutrition: the short nutritional assessment questionnaire (SNAQ). Clin Nutr 2005;24:75-82.

29. Kondrup J, Rasmussen HH, Hamberg O, et al. Nutritional risk screening (NRS 2002): a new method based on an analysis of controlled clinical trials. Clin Nutr 2003;22:321-36.

30. Elia M. Nutritional screening of adults: A multidisciplinary responsibility. Malnutrition Advisory Group (MAG). Redditch: BAPEN, 2003.

31. Rubenstein LZ, Harker JO, Salvà A, et al. Screening for undernutrition in geriatric practice: developing the shortform mini-nutritional assessment (MNA-SF). J Gerontol A Biol Sci Med Sci 2001;56:M366-72.
32. Kovacevich DS, Boney AR, Braunschweig CL, et al. Nutrition risk classification: a reproducible and valid tool for nurses. Nutr Clin Pract 1997;12:20-5.

33. Reilly HM, Martineau JK, Moran A, et al. Nutritional screening--evaluation and implementation of a simple Nutrition Risk Score. Clin Nutr 1995;14:269-73.

34. Guigoz Y, Vellas B, Garry P. Mini nutritional assessment: A practical assessment tool for grading the nutritional state of elderly patients. Facts, Research in Gerontology, 1994:15-59.

35. Veterans Affairs Total Parenteral Nutrition Cooperative Study Group. Perioperative total parenteral nutrition in surgical patients. N Engl J Med 1991;325:525-32.

36. Baker JP, Detsky AS, Wesson DE, et al. Nutritional assessment: a comparison of clinical judgement and objective measurements. N Engl J Med 1982;306:969-72.

37. Cederholm T, Bosaeus I, Barazzoni R, et al. Diagnostic criteria for malnutrition - An ESPEN Consensus Statement. Clin Nutr 2015;34:335-40.

38. van der Gaag NA, Verhaar AC, Haverkort EB, et al. Chylous ascites after pancreaticoduodenectomy: introduction of a grading system. J Am Coll Surg 2008;207:751-7.

39. Rahbari NN, Garden OJ, Padbury R, et al. Posthepatectomy liver failure: a definition and grading by the International Study Group of Liver Surgery (ISGLS). Surgery 2011;149:713-24.

40. Koch M, Garden OJ, Padbury R, et al. Bile leakage after hepatobiliary and pancreatic surgery: a definition and grading of severity by the International Study Group of Liver Surgery. Surgery 2011;149:680-8.

41. Rahbari NN, Garden OJ, Padbury R, et al. Posthepatectomy haemorrhage: a definition and grading by the International Study Group of Liver Surgery (ISGLS). HPB (Oxford) 2011;13:528-35.

42. Dindo D, Demartines N, Clavien PA. Classification of surgical complications: a new proposal with evaluation in a cohort of 6336 patients and results of a survey. Ann Surg 2004;240:205-13.

43. Slankamenac K, Graf R, Barkun J, et al. The comprehensive complication index: a novel continuous scale to measure surgical morbidity. Ann Surg 2013;258:1-7.

44. Team RC. R: A Language and Environment for Statistical Computing. 2013. [cited 2018 28.07.2018]. Available online: http://www.webcitation.org/71FDAlLoh

45. Zacharias T, Ferreira N. Nutritional risk screening 2002 and ASA score predict mortality after elective liver 
resection for malignancy. Arch Med Sci 2017;13:361-9.

46. Ke M, Xu T, Li N, et al. Prognostic nutritional index predicts short-term outcomes after liver resection for hepatocellular carcinoma within the Milan criteria. Oncotarget 2016;7:81611.

47. Buzby GP, Mullen JL, Matthews DC, et al. Prognostic nutritional index in gastrointestinal surgery. Am J Surg 1980;139:160-7.

48. Valero V 3rd, Amini N, Spolverato G, et al. Sarcopenia adversely impacts postoperative complications following resection or transplantation in patients with primary liver tumors. J Gastrointest Surg 2015;19:272-81.

Cite this article as: Probst $\mathrm{P}$, Fuchs J, Schön MR, Polychronidis G, Stravodimos C, Mehrabi A, Diener MK, Knebel P, Büchler MW, Hoffmann K. Prospective study to evaluate the prognostic value of different nutritional assessment scores in liver surgery: NURIMAS Liver (DRKS00006340). HepatoBiliary Surg Nutr 2020;9(4):400-413. doi: 10.21037/ hbsn.2019.06.11
49. Peng PD, van Vledder MG, Tsai S, et al. Sarcopenia negatively impacts short-term outcomes in patients undergoing hepatic resection for colorectal liver metastasis. HPB (Oxford) 2011;13:439-46.

50. Lodewick TM, Roeth AA, Olde Damink SW, et al. Sarcopenia, obesity and sarcopenic obesity: effects on liver function and volume in patients scheduled for major liver resection. J Cachexia Sarcopenia Muscle 2015;6:155-63.

51. Dello SA, Lodewick TM, van Dam RM, et al. Sarcopenia negatively affects preoperative total functional liver volume in patients undergoing liver resection. HPB (Oxford) 2013;15:165-9. 